\title{
InTERFERÊnCIA de HeRbicidas, Associados a UM ANÁlogo de Brassinosteroide, no Aparato Fotossintético de Mudas DE Eucalyptus grandis ${ }^{1}$
}

\author{
Effects of Herbicides, Associated to a Brassinosteroid Analogue, on the Photosynthetic \\ Apparatus of Eucalyptus grandis Seedlings
}

SILVA, C.M.M. ${ }^{2}$, GOMES, M.M.A. ${ }^{3}$ e FREITAS, S.P. ${ }^{4}$

\begin{abstract}
RESUMO - Avaliaram-se os efeitos da aplicação dos herbicidas imazapyr e glyphosate e as suas interações com um análogo espirostânico da castasterona (BB16) no crescimento de mudas clonais de Eucalyptus grandis. Para isso, utilizou-se a aplicação de BB16 (nas concentrações de 0,08 e 0,16 $\mathrm{mg} \mathrm{L}^{-1}$ : BB16(-) e BB16(+), respectivamente), de imazapyr $\left(0,750 \mathrm{~kg} \mathrm{ha}^{-1}\right)$ e de glyphosate $\left(1,440 \mathrm{~kg} \mathrm{ha}^{-1}\right)$. Os tratamentos foram constituídos pelo controle, BB16(-), BB16(+), glyphosate, imazapyr, além da interação hormônio-herbicida, em intervalo de 12 h entre a aplicação de BB16 e de cada herbicida, e vice-versa. A mensuração da fluorescência da clorofila $a$ foi realizada diariamente, na terceira folha totalmente expandida, além das avaliações de altura, diâmetro e biomassa seca da parte aérea (BSPA) e das raízes (BSR). O controle, BB16(-) e BB16(+) diferiram dos demais tratamentos para BSPA, porém, para BSR, altura e diâmetro, não houve diferença significativa entre os tratamentos. As médias dos parâmetros de fluorescência, obtidas para os tratamentos que receberam a aplicação dos herbicidas, foram superiores ao controle, BB16(-) e BB16(+) até o sétimo dia após a aplicação dos tratamentos (DAT). As mudas que receberam aplicação de glyphosate este associado, ou não, a BB16(-) ou BB16(+) - apresentaram-se necróticas antes do sétimo DAT, enquanto as que receberam imazapyr associado ao BB16 manifestaram apenas as extremidades dos ramos laterais necrosadas. Os herbicidas testados causaram efeitos deletérios no aparato fotossintético de E. grandis; todavia, a partir do sétimo DAT as mudas que receberam a aplicação de imazapyr (com ou sem BB16) não diferiram dos tratamentos controle, BB16(-) e BB16(+), quanto aos parâmetros de fluorescência.
\end{abstract}

Palavras-chave: BB16, glyphosate, imazapyr, interação homônio-herbicidas.

\begin{abstract}
The effects of the application of imazapyr and glyphosate and their interactions with a spirostanic analogue of castasterone (BB16), on the growth of seedling clones of Eucalyptus grandis were evaluated. Applications of BB16 (at concentrations of 0.08 and $0.16 \mathrm{mg} \mathrm{L}^{-1}: B B 16(-)$ and BB16 (+) respectively), and of imazapyr $(0.750 \mathrm{~kg} \mathrm{ha-1})$ and glyphosate $\left(1.440 \mathrm{~kg} \mathrm{ha} \mathrm{h}^{-1}\right)$ were carried out. The treatments were the control, BB16 (-), BB16 (+), glyphosate, imazapyr, besides the hormone-herbicide interaction, at an interval of $12 \mathrm{~h}$ between the BB1 6 and each herbicide application and vice versa. Chlorophyll a fluorescence was measured daily on the third completely - expanded leaf, as well as height, diameter and dry matter of the shoot (DMS) and roots (DMR). The control, BB16 (-) and BB16(+) differed from the other DMS treatments; however, for DMR, height and diameter did not show any significant difference among the treatments. The fluorescence parameter means obtained for the herbicide application treatments were higher than the control, BB16 (-) and BB16 (+) until the seventh day after treatment (DAT). The seedlings that received the glyphosate application associated, or not, to $B B 16(-)$ or $B B 16(+)$, exhibited necrosis
\end{abstract}

Recebido para publicação em 23.10.2008 e na forma revisada em 13.11.2009.

2 D.Sc. em Produção Vegetal, <carlos.magno@rjo.incra.gov.br>; ${ }^{3}$ Profa. da Fundação de Apoio à Escola Técnica do Estado do Rio de Janeiro, Av. Wilson Batista, s/n, Parque Aldeia, 28000-000 Campos dos Goytacazes-RJ; ${ }^{4}$ Professor do Laboratório de Fitotecnia do Centro de Ciências e Tecnologias Agropecuárias da Universidade Estadual do Norte Fluminense "Darcy Ribeiro", Av. Alberto Lamego, 2000, 28013-602 Campos dos Goytacazes-RJ.

Planta Daninha, Viçosa-MG, v. 27, n. 4, p. 789-797, 2009 
before the seventh day, while the seedlings that received imazapyr associated to BB16 showed only necrotic lesion at the extremity of the lateral branches. The herbicides analyzed caused deleterious effects on the photosynthetic apparatus of $\boldsymbol{E}$. grandis. However, since the seventh $D A T$, the seedlings that received the imazapyr application (with or without BB16) did not differ from the control, $B B 16(-)$ and $B B 16(+)$ treatments, in relation to the fluorescence parameters.

Keywords: BB16, glyphosate, hormone-herbicide interaction, imazapyr.

\section{INTRODUÇÃO}

No processo fotossintético, a luz é absorvida por pigmentos do complexo-antena, que, excitados, transferem energia para os centros de reação dos fotossistemas I e II $\left(\mathrm{P}_{700}\right.$ e $\mathrm{P}_{680}$, respectivamente) (Young \& Frank, 1996). Quando ocorre excesso de energia, esta pode ser dissipada na forma de fluorescência da clorofila (Krause \& Winter, 1996), sendo essa uma das formas de monitoramento da inibição ou redução na transferência de elétrons entre os fotossistemas da planta sob estresse. A redução na dissipação da energia pelo processo fotoquímico é refletida por incremento correspondente na fluorescência, a qual pode ser observada ainda em folhas intactas (Maxwell \& Johnson, 2000).

Há evidências de que muitos inibidores de processos metabólicos, que não estão diretamente ligados ao processo fotossintético, podem causar modificações na cinética da fluorescência (Christensen et al., 2003; Xia et al., 2006). Em função da aplicação de herbicidas, mesmo quando o sintoma ainda não é visivel, ocorrem injúrias causadas ao aparelho fotossintético, reduzindo a capacidade de realização de fotossíntese pela planta (Barbagallo et al., 2003); essa alteração pode ser avaliada através da análise da fluorescência da clorofila a (Catunda et al., 2005). O glyphosate, por exemplo, interrompe a síntese de aminoácidos aromáticos por bloquear a rota do ácido xiquímico, induzindo significativas alterações na emissão de fluorescência (Ireland et al., 1986), associada às modificações no metabolismo do carbono fotossintético (Madsen et al., 1995).

Dessa forma, a inibição de reações metabólicas não envolvidas diretamente na fotossintese pode modificar a sintese de intermediários utilizados no processo fotossintético e, assim, interferir na fotossíntese e na emissão de fluorescência (Baker \& Rosenqvist, 2004).

A mensuração de fluorescência é um método rápido, simples e não invasivo, sendo largamente utilizada no entendimento dos mecanismos da fotossintese, bem como na avaliação da capacidade fotossintética alterada por estresses bióticos ou abióticos pelos quais as plantas possam passar. Nesse tipo de avaliação são utilizados fluorômetros de luz modulada (Schreiber et al., 1996), cujas aplicações variam desde a rápida identificação de injúrias causadas ao aparelho fotossintético, até a análise detalhada da alteração da capacidade fotossintética da planta.

Utilizando um fluorômetro de luz modulada, obtêm-se os valores de $F_{o}$, que representa a fluorescência inicial correspondente à fração da energia absorvida pelo complexo-antena e não transmitida, portanto, não absorvida pelos pigmentos fotossintéticos (Rascher et al., 2000); de $F_{m}$, que é a fluorescência máxima; e da relação $F_{v} / F_{m}$, que representa o rendimento quântico máximo do fotossistema II (FSII). A relação $F_{v} / F_{m}$ pode variar de 0,75 a 0,85 em plantas não submetidas a estresses (Bolhàr-Nordenkamph et al., 1989), sendo a redução dessa razão um excelente indicador de efeito fotoinibitório quando as plantas estão submetidas ao estresse químico (Araus \& Hogan, 1994).

É sabido que os brassinosteroides, de forma geral, participam efetivamente de processos de tolerância das plantas a diversos tipos de estresse, como temperaturas extremas, seca, salinidade e ataque de patógenos (Krishna, 2003), principalmente em virtude da indução de enzimas antioxidantes (Özdemir et al., 2004). Assim, é possivel também que essas substâncias possam conferir tolerância aos 
estresses químicos, como os advindos da aplicação de herbicidas (Xia et al., 2006), funcionando como antídoto à presença destes.

Objetivando avaliar os efeitos no aparato fotossintético e no crescimento de mudas de Eucalyptus grandis, em decorrência da aplicação dos herbicidas imazapyr (inibidor da enzima acetolactato sintase) e glyphosate (inibidor da enzima 3-enoilpiruvilxiquimato5-fosfato sintase), e as suas interações com um análogo de brassinosteroide, foi realizado um ensaio em condições de casa de vegetação.

\section{MATERIAL E MÉTODOS}

O experimento foi instalado em sistema hidropônico, utilizando vasos plásticos com 2,5 L de solução nutritiva (Clark, 1975) aerada ininterruptamente, com correção do $\mathrm{pH}$ a cada dois dias. Os vasos receberam revestimento prateado nas paredes externas e foram envoltos internamente por camada plástica transparente.

As mudas de eucalipto utilizadas foram provenientes de propagação vegetativa, com 105 dias de idade e altura média de $32,8 \mathrm{~cm}$, produzidas em tubetes de $55 \mathrm{~cm}^{3}$ de capacidade. Quando do transplante, utilizaram-se mudas homogêneas em altura, as quais foram transferidas para solução nutritiva, sendo uma muda por vaso, onde permaneceram por 60 dias, para emissão de novas folhas e raízes. A solução foi trocada semanalmente, sendo a última no dia da aplicação foliar dos compostos, a qual foi realizada com pulverizador costal pressurizado a $\mathrm{CO}_{2}$, com $30 \mathrm{lb} \mathrm{pol}^{-2}$ e volume de calda de $200 \mathrm{~L} \mathrm{ha}^{-1}$, equipado com um bico de jato plano (tipo leque) Teejet DG95.02 EVS, tendo-se o cuidado prévio de cobrir a superficie dos vasos com dupla camada plástica, para evitar a contaminação da solução nutritiva.

Os compostos exógenos aplicados foram constituídos pelos herbicidas glyphosate $(G)$ e imazapyr (I), nas doses de 1,440 e $0,750 \mathrm{~kg} \mathrm{ha}^{-1}$ do ingrediente ativo, respectivamente, além da aplicação do hormônio BB16 (BIOBRAS-16, análogo espirostânico da castasterona - (25R)$2 \alpha, 3 \alpha$-diidroxi- $5 \alpha$-espirostan- 6 -ona (Coll et al., 1995)), este em duas concentrações $(0,08$ e $\left.0,16 \mathrm{mg} \mathrm{L}^{-1}\right)$, simbolizadas por BB16(-) e $\mathrm{BB} 16(+)$, respectivamente.
O ensaio consistiu da avaliação dos efeitos dos tratamentos controle (sem a aplicação de compostos exógenos), da aplicação de BB16(-), BB16(+), G, I, além da interação hormônioherbicida. A aplicação desses compostos foi feita em intervalos de 12 horas, o que configura a aplicação de glyphosate ou imazapyr 12 horas após a de BB16 (em ambas as concentrações), e vice-versa.

O monitoramento da fluorescência foi realizado diariamente, até o quinto dia após a aplicação dos tratamentos somente com glyphosate ou este associado ao hormônio BB16, e até o $11^{\circ}$ dia para os demais tratamentos, às 16 horas em temperatura ambiente, tomandose a medida da terceira folha totalmente expandida (da parte apical para a base da muda).

A folha medida foi pré-adaptada ao escuro, com a utilização de pinças, por 30 minutos antes das determinações de fluorescência. Após esse período, a iluminação foi fornecida por meio de um conjunto de 6 LEDs com comprimento de onda de $650 \mathrm{~nm}$ na superficie da amostra, para fornecer energia luminosa homogênea de $3.000 \mu$ moles $\mathrm{m}^{-2} \mathrm{~s}^{-1}$, em $100 \%$ da intensidade luminosa, sob uma área de $4 \mathrm{~mm}$ de diâmetro. Por meio de um fluorômetro de luz não modulada, modelo PEA (Hansatech Instruments Ltd, King's Lynn, Norfolk, UK), foi possivel obter as variáveis $F_{O}$ (fluorescência inicial), $F_{m}$ (fluorescência máxima) e a relação $F_{v} / F_{m}$ (eficiência fotoquímica do FSII).

Utilizando-se do programa computacional Biolyzer (R.J. Strasser, University of Geneva, Laboratory of Bioenergetics, Switzerland), foram obtidos alguns indicadores do desempenho do processo fotossintético, como o ABS/RC (referente à quantidade de energia absorvida pelos pigmentos no complexo-antena do FSII, por unidade de centro de reação ativo) (Strasser et al., 2000).

Ao final do período experimental (12 dias após a aplicação dos tratamentos - DAT) foram aferidos o diâmetro (tomado a $2,5 \mathrm{~cm}$ do colo da muda) e a altura (esta relativa à distância entre o colo e a inserção da folha mais nova no ápice da muda). Determinaram-se também as biomassas secas de parte aérea e do sistema radicular após coleta das mudas e foi feita a secção do caule à altura da inserção das raízes, com acondicionamento das partes das 
mudas, separadamente, em sacos de papel, que foram levadas à estufa com circulação de ar, a $72 \pm 1{ }^{\circ} \mathrm{C}$, por aproximadamente 72 horas

O delineamento estatístico utilizado foi inteiramente casualizado, com 13 tratamentos e quatro repetições; os dados obtidos foram submetidos à análise de variância.

\section{RESULTADOS E DISCUSSÃO}

Somente as plantas que receberam a aplicação exclusiva do análogo de brassinosteroide (BB16), quer na concentração de $0,08 \mathrm{mg} \mathrm{L}^{-1}$ ou $0,16 \mathrm{mg} \mathrm{L}^{-1}$, não apresentaram redução de biomassa seca da parte aérea, uma vez que não diferiram do tratamento controle. Comportamento semelhante foi observado para a variável biomassa seca total. Contudo, os tratamentos constituídos pela aplicação de BB16 (em ambas as concentrações), anteriormente aos herbicidas ou posteriormente à aplicação de glyphosate, resultaram em decréscimo significativo da biomassa seca de raízes (Figura 1). Já com relação às variáveis biométricas (altura e diâmetro), não se verificou diferença entre os tratamentos.

Mesmo não apresentando diferenças de intoxicação mediante a análise de variáveis biométricas, puderam ser verificados comportamentos distintos entre os tratamentos ao se analisarem os parâmetros da fluorescência (Figuras 2 e 3).

Independentemente do parâmetro avaliado, a partir do quinto dia, em virtude da desidratação da folha-medida, não foi possível fazer mensurações do aparato fotossintético no tratamento que recebeu a aplicação de glyphosate e, 12 horas após, a de BB16; o mesmo ocorreu no sexto dia para os tratamentos constituídos pela aplicação de BB16 anteriormente à de glyphosate e, no sétimo dia, para o tratamento somente com glyphosate (Figura 2).

As plantas que receberam a aplicação de BB16(+) após a de imazapyr apresentaram significativo efeito deletério ao aparato fotossintético até o quinto DAT, uma vez que se
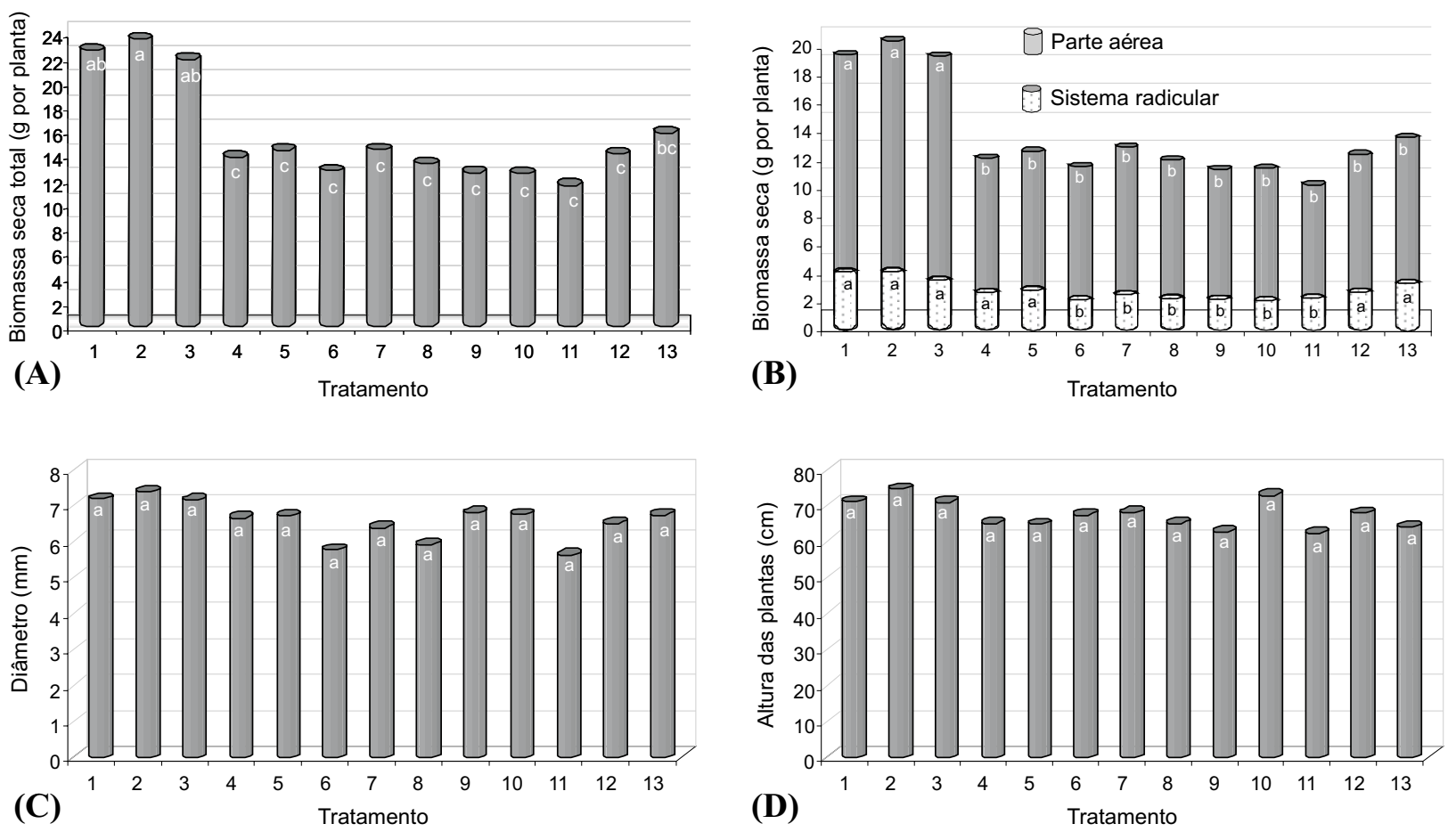

Figura 1 - Biomassa seca total (A), da parte aérea e do sistema radicular (B), diâmetro (C) e altura (D) de mudas de Eucalyptus grandis aos 12 DAT, em função dos tratamentos: 1-testemunha; 2-BB16(-); 3-BB16(+); 4 glyphosate; 5-imazapyr; 6-BB16(-)+G; 7BB16(-)+I; 8-BB16(+)+G; 9-BB16(+)+I; 10-G+BB16(-); 11-G+BB16(+); 12-I+BB16(-);13-I+BB16(+). Médias seguidas de letras iguais, dentro de cada variável analisada, não diferem entre si pelo teste de Duncan a $5 \%$ de probabilidade. 
pode verificar que o valor da razão $A B S / R C$ foi maior para as plantas deste tratamento (Figura 2). Isso ocorreu devido à destruição dos centros de reação ativos, representados pelos menores valores de RC. Contudo, a partir do sexto DAT ele não diferiu dos demais tratamentos.

O valor da fluorescência inicial $\left(F_{0}\right)$ pode ser elevado caso os centros de reação do FSII estejam comprometidos ou se a transferência da energia de excitação do complexo-antena para os centros de reação estiver prejudicada (Bolhàr-Nordenkamph et al., 1989). Para os tratamentos imazapyr (I) e I+BB16(+), verificou-se que os elevados valores de $F_{O}$ até o sexto DAT (Figura 3A) representaram alto nível de fluorescência emanada quando a quinona $a\left(Q_{a}\right)$ estava no seu estado de máxima oxidação, portanto, quando os centros de reação do FSII estavam totalmente abertos (Baker \& Rosenqvist, 2004). Nessa situação, quanto maior o valor de $F_{o}$, maior será a tentativa dos fotosssistemas em dissipar, na forma de fluorescência, o excesso de energia não aproveitado.

Ao comparar as curvas de fluorescência máxima (Figura 3B), não se verificou diferença estatística (Tabela 1) entre os tratamentos para esse parâmetro no final do periodo experimental; os que receberam a aplicação de glyphosate não persistiram até a última avaliação, dada a morte das mudas nos primeiros dias de avaliação.

Elevados valores de $F_{v} / F_{m}(0,75$ a 0,85$)$ refletem adequada taxa de conversão de energia luminosa em fluxo de elétrons no fotossistema II. Nesses casos, necessita-se de eficiente estruturação do FSII, a qual é obtida mediante quantidade considerável de moléculas de clorofilas não degradadas, e ordenação dos polipeptídeos que compõem esse fotossistema (Bolhàr-Nordenkamph et al., 1989). Ao analisar essa relação, pôde-se verificar que, a partir do quarto DAT, as mudas que receberam a aplicação dos herbicidas (precedidos ou não da aplicação de BB16) apresentaram relação $F_{v} / F_{m}$ abaixo de 0,75 , o que indica que houve significativa redução na sua taxa de conversão de energia luminosa (Figura 3C). Já para os tratamentos que receberam glyphosate (antes ou após esse análogo hormonal) não foi possivel obter os valores de $F_{v} / F_{m}$ a partir do quinto DAT (Figura $3 \mathrm{C}$ ), em razão da necrose da folha-medida.

Nos tratamentos compostos pela aplicação dos herbicidas, obtiveram-se médias de rendimento quântico máximo do FSII inferiores às das mudas controle e àquelas

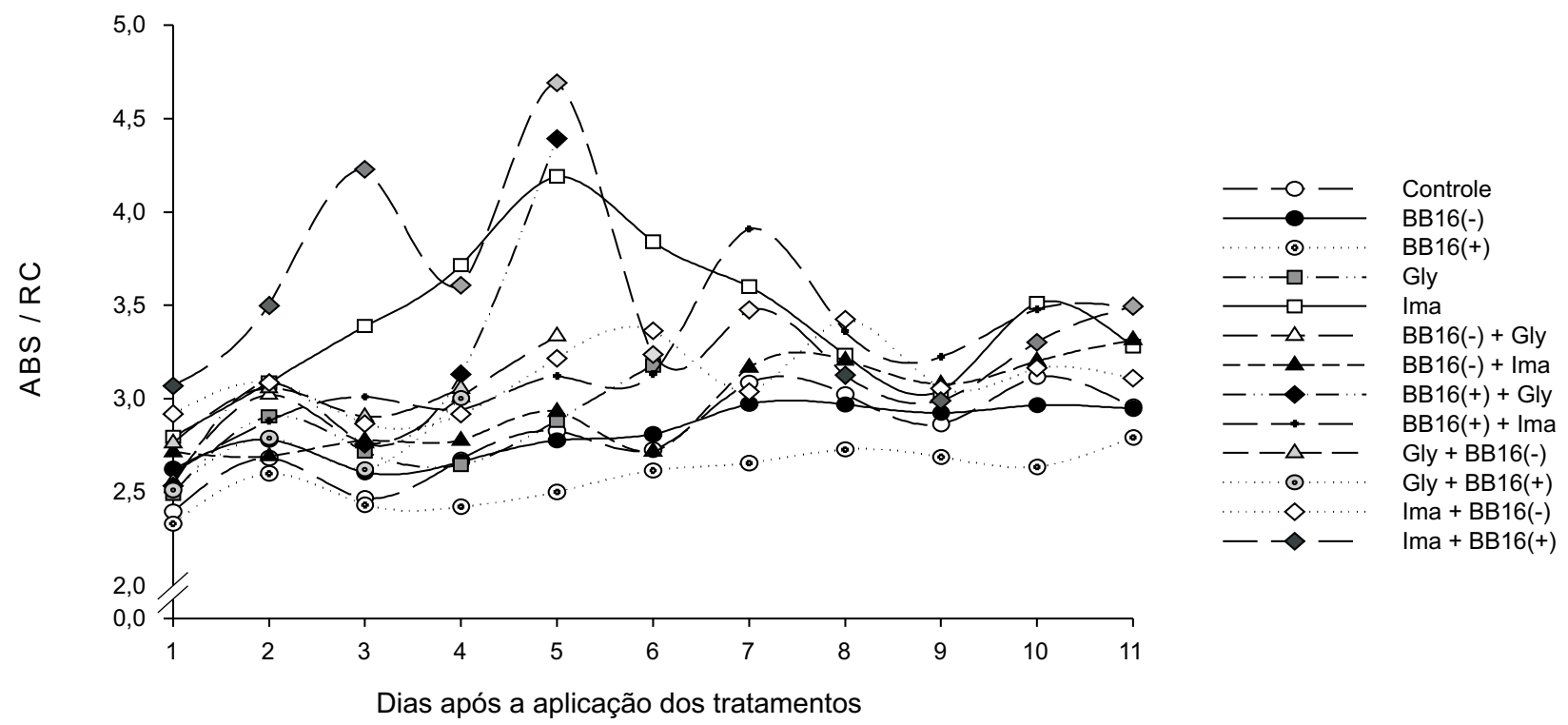

Figura 2 - Valores da energia absorvida por unidade de centro de reação ativo (ABS/RC), mensurada às 16 h, ao longo do período experimental, em mudas de Eucalyptus grandis. 
que receberam aplicação somente de BB16; nestas últimas, o valor da relação $F_{v} / F_{m}$ foi superior a 0,75 ao longo do ensaio (Figura 3C), sugerindo que os herbicidas tenham provocado redução no fluxo de elétrons entre os fotossistemas, com consequente redução na quantidade de energia aproveitada pela planta para a realização dos processos fotoquímicos, como fixação de $\mathrm{CO}_{2}$ e redução de NADPH.

Verificou-se que, quando se utilizou somente a aplicação de glyphosate, ocorreu

Tabela 1 - Médias diárias da eficiência quântica do fotossistema II (FSII) (ABS/RC), da fluorescência inicial $\left(F_{0}\right)$ e máxima $\left(F_{m}\right)$ e do rendimento quântico máximo do FSII $\left(F_{v} / F_{m}\right)$ de plantas de eucalipto, submetidas a compostos exôgenos

\begin{tabular}{|c|c|c|c|c|c|c|c|c|c|c|c|c|c|c|c|}
\hline Var. & DAT $^{1}$ & Controle & BB16(-) & $\mathrm{BB} 16(+)$ & $\mathrm{G}$ & I & BB16(-)+G & BB16(-)+I & BB16(+)+G & $\mathrm{BB} 16(+)+\mathrm{I}$ & G+BB16(-) & $\mathrm{G}+\mathrm{BB} 16(+)$ & $\mathrm{I}+\mathrm{BB} 16(-)$ & $\mathrm{I}+\mathrm{BB} 16(+)$ & $\mathrm{CV}$ \\
\hline \multirow{11}{*}{ 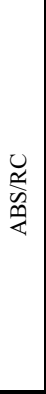 } & 1 & $2,40 \mathrm{a}$ & $2,63 \mathrm{a}$ & $2,33 \mathrm{a}$ & $2,49 \mathrm{a}$ & $2,80 \mathrm{a}$ & $2,56 \mathrm{a}$ & $2,71 \mathrm{a}$ & $2,54 \mathrm{a}$ & $2,61 \mathrm{a}$ & $2,76 \mathrm{a}$ & $2,51 \mathrm{a}$ & $2,92 \mathrm{a}$ & $3,07 \mathrm{a}$ & 12,8 \\
\hline & 2 & $2,68 \mathrm{~b}$ & $2,78 \mathrm{~b}$ & $2,60 \mathrm{~b}$ & $2,91 \mathrm{~b}$ & $3,09 \mathrm{~b}$ & $3,02 \mathrm{~b}$ & $2,69 \mathrm{~b}$ & $3,08 \mathrm{~b}$ & $2,88 \mathrm{~b}$ & $3,06 \mathrm{~b}$ & $2,79 \mathrm{~b}$ & $3,09 \mathrm{~b}$ & $3,50 \mathrm{a}$ & 11,5 \\
\hline & 3 & $2,47 \mathrm{~b}$ & $2,61 \mathrm{~b}$ & $2,43 \mathrm{~b}$ & $2,72 \mathrm{~b}$ & $3,39 \mathrm{a}$ & $2,76 b$ & $2,78 \mathrm{~b}$ & $2,76 \mathrm{~b}$ & $3,01 \mathrm{~b}$ & $2,90 \mathrm{~b}$ & $2,62 \mathrm{~b}$ & $2,87 \mathrm{~b}$ & $4,23 \mathrm{a}$ & 13,3 \\
\hline & 4 & $2,68 \mathrm{bc}$ & $2,66 \mathrm{bc}$ & $2,42 \mathrm{c}$ & $2,65 \mathrm{bc}$ & $3,72 \mathrm{a}$ & $3,01 \mathrm{bc}$ & $2,78 \mathrm{bc}$ & $3,13 \mathrm{bc}$ & $2,94 \mathrm{bc}$ & $3,06 \mathrm{bc}$ & $3,00 \mathrm{bc}$ & $2,92 \mathrm{bc}$ & $3,61 \mathrm{a}$ & 13,1 \\
\hline & 5 & $2,83 \mathrm{ab}$ & $2,78 \mathrm{ab}$ & $2,50 \mathrm{~b}$ & $2,88 \mathrm{ab}$ & $4,19 \mathrm{a}$ & $3,34 \mathrm{ab}$ & $2,93 \mathrm{ab}$ & $4,39 \mathrm{a}$ & $3,12 \mathrm{ab}$ & & & $3,22 \mathrm{ab}$ & $4,69 \mathrm{a}$ & 23,4 \\
\hline & 6 & $2,73 \mathrm{~b}$ & $2,81 \mathrm{~b}$ & $2,62 \mathrm{~b}$ & $3,18 \mathrm{ab}$ & $3,84 \mathrm{ab}$ & & $2,72 \mathrm{~b}$ & & $3,13 \mathrm{ab}$ & & & $3,36 \mathrm{ab}$ & $3,24 \mathrm{a}$ & 10,7 \\
\hline & 7 & $3,09 \mathrm{a}$ & $2,97 \mathrm{a}$ & $2,66 \mathrm{a}$ & & $3,60 \mathrm{a}$ & & $3,17 \mathrm{a}$ & & 3,91 a & & & $3,04 \mathrm{a}$ & $3,48 \mathrm{a}$ & 19,6 \\
\hline & 8 & $3,02 \mathrm{a}$ & $2,97 \mathrm{a}$ & $2,73 \mathrm{a}$ & & $3,23 \mathrm{a}$ & & $3,21 \mathrm{a}$ & & $3,36 \mathrm{a}$ & & & $3,43 \mathrm{a}$ & $3,13 \mathrm{a}$ & 21,1 \\
\hline & 9 & $2,86 \mathrm{a}$ & $2,93 \mathrm{a}$ & $2,69 \mathrm{a}$ & & $3,04 \mathrm{a}$ & & $3,08 \mathrm{a}$ & & $3,23 \mathrm{a}$ & & & $3,05 \mathrm{a}$ & $2,99 \mathrm{a}$ & 10,6 \\
\hline & 10 & $3,12 \mathrm{a}$ & $2,97 \mathrm{a}$ & $2,64 \mathrm{a}$ & & $3,51 \mathrm{a}$ & & $3,20 \mathrm{a}$ & & $3,48 \mathrm{a}$ & & & $3,16 \mathrm{a}$ & $3,30 \mathrm{a}$ & 13,1 \\
\hline & 11 & $2,96 \mathrm{a}$ & $2,95 \mathrm{a}$ & $2,79 \mathrm{a}$ & & $3,28 \mathrm{a}$ & & $3,31 \mathrm{a}$ & & $3,49 \mathrm{a}$ & & & $3,11 \mathrm{a}$ & $3,50 \mathrm{a}$ & 12,5 \\
\hline \multirow{11}{*}{ 送 } & 1 & 594,3 a & $594,8 \mathrm{a}$ & $528,0 \mathrm{a}$ & $609,8 \mathrm{a}$ & $663,3 \mathrm{a}$ & $587,5 \mathrm{a}$ & 639,3 a & $563,5 \mathrm{a}$ & $635,3 \mathrm{a}$ & $626,8 \mathrm{a}$ & $561,5 \mathrm{a}$ & $737,3 \mathrm{a}$ & 789,3 a & 21,1 \\
\hline & 2 & $535,8 \mathrm{~b}$ & $565,8 \mathrm{~b}$ & $530,8 \mathrm{~b}$ & $590,0 \mathrm{~b}$ & $715,3 \mathrm{ab}$ & $659,8 \mathrm{ab}$ & $582,0 \mathrm{~b}$ & $632,3 \mathrm{ab}$ & $610,0 \mathrm{~b}$ & $607,8 \mathrm{~b}$ & $565,0 \mathrm{~b}$ & $709,3 \mathrm{ab}$ & $847,5 \mathrm{a}$ & 13,8 \\
\hline & 3 & $512,5 \mathrm{c}$ & $554,3 \mathrm{c}$ & $515,3 \mathrm{c}$ & $588,0 \mathrm{bc}$ & $907,3 \mathrm{ab}$ & $611,5 \mathrm{bc}$ & $666,3 \mathrm{bc}$ & $617,8 \mathrm{bc}$ & $728,8 \mathrm{bc}$ & $587,8 \mathrm{bc}$ & $564,3 \mathrm{c}$ & $699,0 \mathrm{bc}$ & $1099,0 \mathrm{a}$ & 20,1 \\
\hline & 4 & $566,5 \mathrm{c}$ & $590,5 \mathrm{bc}$ & $535,0 \mathrm{c}$ & $605,3 \mathrm{bc}$ & $1020,5 \mathrm{a}$ & $711,8 \mathrm{abc}$ & $661,8 \mathrm{bc}$ & $717,3 \mathrm{abc}$ & $683,8 \mathrm{bc}$ & $636,5 \mathrm{bc}$ & $650,3 \mathrm{bc}$ & $696,7 \mathrm{abc}$ & $905,3 \mathrm{a}$ & 18,4 \\
\hline & 5 & $590,3 \mathrm{bc}$ & $599,3 \mathrm{bc}$ & $538,3 \mathrm{c}$ & $672,7 \mathrm{bc}$ & $1105,0 \mathrm{a}$ & $740,3 \mathrm{abc}$ & $732,3 \mathrm{abc}$ & $781,3 \mathrm{abc}$ & $788,0 \mathrm{abc}$ & & & $830,3 \mathrm{abc}$ & $997,5 \mathrm{ab}$ & 21,5 \\
\hline & 6 & $592,8 \mathrm{~b}$ & $636,8 \mathrm{~b}$ & $574,5 \mathrm{~b}$ & $734,7 \mathrm{~b}$ & $1164,8 \mathrm{~b}$ & & $634,0 \mathrm{~b}$ & & $806,5 \mathrm{~b}$ & & & $828,3 \mathrm{ab}$ & $814,5 \mathrm{a}$ & 17,5 \\
\hline & 7 & $673,8 \mathrm{a}$ & $659,3 \mathrm{a}$ & $590,0 \mathrm{a}$ & & $1081,7 \mathrm{a}$ & & $757,3 \mathrm{a}$ & & $1020,3 \mathrm{a}$ & & & $768,0 \mathrm{a}$ & $888,5 \mathrm{a}$ & 29,9 \\
\hline & 8 & $659,0 \mathrm{a}$ & $642,5 \mathrm{a}$ & $572,5 \mathrm{a}$ & & $1043,3 \mathrm{a}$ & & 768,7 a & & $954,5 \mathrm{a}$ & & & $864,3 \mathrm{a}$ & $716,5 \mathrm{a}$ & 27,6 \\
\hline & 9 & $593,5 \mathrm{a}$ & $625,0 \mathrm{a}$ & $558,8 \mathrm{a}$ & & $821,0 \mathrm{a}$ & & $713,7 \mathrm{a}$ & & 795,7 a & & & $662,3 \mathrm{a}$ & $701,0 \mathrm{a}$ & 19,2 \\
\hline & 10 & $652,0 \mathrm{a}$ & $627,5 \mathrm{a}$ & $549,5 \mathrm{a}$ & & 909,0 a & & $772,0 \mathrm{a}$ & & 867,5 a & & & $760,5 \mathrm{a}$ & $732,5 \mathrm{a}$ & 23,2 \\
\hline & 11 & $609,8 \mathrm{a}$ & $613,8 \mathrm{a}$ & $584,5 \mathrm{a}$ & & $903,0 \mathrm{a}$ & & 799,7 a & & $927,3 \mathrm{a}$ & & & $654,3 \mathrm{a}$ & $788,5 \mathrm{a}$ & 23,2 \\
\hline \multirow{11}{*}{$x^{E}$} & 1 & $3696 \mathrm{a}$ & $3585 \mathrm{a}$ & $3556 \mathrm{a}$ & 3493 a & $3457 \mathrm{a}$ & $3490 \mathrm{a}$ & $3443 \mathrm{a}$ & $3657 \mathrm{a}$ & $3560 \mathrm{a}$ & 3413 a & $3512 \mathrm{a}$ & $3379 \mathrm{a}$ & $3422 \mathrm{a}$ & 6,2 \\
\hline & 2 & $3155 \mathrm{a}$ & $3343 \mathrm{a}$ & $3359 \mathrm{a}$ & $2970 \mathrm{a}$ & $3139 \mathrm{a}$ & $3172 \mathrm{a}$ & $2935 \mathrm{a}$ & $3171 \mathrm{a}$ & $3036 \mathrm{a}$ & $3018 \mathrm{a}$ & $3157 \mathrm{a}$ & $2814 \mathrm{a}$ & $2825 \mathrm{a}$ & 8,4 \\
\hline & 3 & $3296 \mathrm{a}$ & $3533 \mathrm{a}$ & $3528 \mathrm{a}$ & $3084 \mathrm{a}$ & $3432 \mathrm{a}$ & $3314 \mathrm{a}$ & $3349 \mathrm{a}$ & $3281 \mathrm{a}$ & $3370 \mathrm{a}$ & $3227 \mathrm{a}$ & $3068 \mathrm{a}$ & $3344 \mathrm{a}$ & $2859 \mathrm{a}$ & 8,5 \\
\hline & 4 & $3251 \mathrm{a}$ & $3319 \mathrm{a}$ & $3217 \mathrm{a}$ & $3076 \mathrm{a}$ & $3118 \mathrm{a}$ & $3131 \mathrm{a}$ & $3246 \mathrm{a}$ & $2760 \mathrm{a}$ & $3059 \mathrm{a}$ & $2799 \mathrm{a}$ & $2836 \mathrm{a}$ & $3373 \mathrm{a}$ & $3116 \mathrm{a}$ & 10,0 \\
\hline & 5 & $3176 \mathrm{a}$ & $3324 \mathrm{a}$ & $3247 \mathrm{a}$ & $2952 \mathrm{a}$ & $2869 \mathrm{a}$ & $2845 \mathrm{a}$ & $3073 \mathrm{a}$ & $2058 \mathrm{~b}$ & $3136 \mathrm{a}$ & & & $3187 \mathrm{a}$ & $2805 \mathrm{a}$ & 16,7 \\
\hline & 6 & $3131 \mathrm{a}$ & $3395 \mathrm{a}$ & $3247 \mathrm{a}$ & $2676 \mathrm{a}$ & $3399 \mathrm{a}$ & & $3220 \mathrm{a}$ & & $3268 \mathrm{a}$ & & & $3549 \mathrm{a}$ & $3236 \mathrm{a}$ & 10,6 \\
\hline & 7 & $2857 \mathrm{ab}$ & $3158 \mathrm{a}$ & $2893 \mathrm{ab}$ & & $3339 \mathrm{a}$ & & $2582 \mathrm{~b}$ & & $3018 \mathrm{ab}$ & & & $3321 \mathrm{a}$ & $3098 \mathrm{ab}$ & 6,7 \\
\hline & 8 & $2973 \mathrm{a}$ & $3276 \mathrm{a}$ & $3159 \mathrm{a}$ & & 3047 a & & $2815 \mathrm{a}$ & & $3260 \mathrm{a}$ & & & $3296 \mathrm{a}$ & $3056 \mathrm{a}$ & 11,4 \\
\hline & 9 & $3103 \mathrm{a}$ & $3239 \mathrm{a}$ & $3200 \mathrm{a}$ & & $3460 \mathrm{a}$ & & $2930 \mathrm{a}$ & & $3206 \mathrm{a}$ & & & $3200 \mathrm{a}$ & $3061 \mathrm{a}$ & 7,4 \\
\hline & 10 & $2943 \mathrm{a}$ & $3170 \mathrm{a}$ & $3064 \mathrm{a}$ & & $3442 \mathrm{a}$ & & $2928 \mathrm{a}$ & & $3156 \mathrm{a}$ & & & $3250 \mathrm{a}$ & $2860 \mathrm{a}$ & 6,6 \\
\hline & 11 & $3006 \mathrm{~b}$ & $3170 \mathrm{ab}$ & $3079 \mathrm{ab}$ & & $3441 \mathrm{a}$ & & $2938 \mathrm{~b}$ & & $3319 \mathrm{ab}$ & & & $2999 \mathrm{~b}$ & 2976 b & 6,6 \\
\hline \multirow{11}{*}{$x^{2}$} & 1 & $0,799 \mathrm{ab}$ & $0,792 \mathrm{ab}$ & $0,811 \mathrm{a}$ & $0,782 \mathrm{ab}$ & $0,742 \mathrm{ab}$ & $0,789 \mathrm{ab}$ & $0,760 \mathrm{ab}$ & $0,800 \mathrm{ab}$ & $0,772 \mathrm{ab}$ & $0,767 \mathrm{ab}$ & $0,794 \mathrm{ab}$ & $0,740 \mathrm{ab}$ & $0,706 \mathrm{~b}$ & 4,8 \\
\hline & 2 & $0,787 \mathrm{ab}$ & $0,786 \mathrm{ab}$ & 0,799 a & $0,746 \mathrm{abc}$ & $0,703 \mathrm{abc}$ & $0,738 \mathrm{abc}$ & $0,738 \mathrm{abc}$ & $0,747 \mathrm{abc}$ & $0,734 \mathrm{abc}$ & $0,743 \mathrm{abc}$ & $0,773 \mathrm{ab}$ & $0,677 \mathrm{bc}$ & $0,636 \mathrm{c}$ & 6,4 \\
\hline & 3 & $0,801 \mathrm{ab}$ & $0,800 \mathrm{ab}$ & $0,811 \mathrm{a}$ & $0,754 \mathrm{ab}$ & $0,675 \mathrm{bc}$ & $0,767 \mathrm{ab}$ & $0,739 \mathrm{ab}$ & $0,769 \mathrm{ab}$ & $0,724 \mathrm{ab}$ & $0,764 \mathrm{ab}$ & $0,766 \mathrm{ab}$ & $0,727 \mathrm{ab}$ & $0,562 \mathrm{c}$ & 7,0 \\
\hline & 4 & $0,785 \mathrm{ab}$ & $0,783 \mathrm{ab}$ & $0,797 \mathrm{a}$ & $0,757 \mathrm{abc}$ & $0,613 \mathrm{c}$ & $0,727 \mathrm{abc}$ & $0,733 \mathrm{abc}$ & $0,699 \mathrm{abc}$ & $0,709 \mathrm{abc}$ & $0,718 \mathrm{abc}$ & $0,727 \mathrm{abc}$ & 0,729 abc & $0,645 \mathrm{bc}$ & 7,8 \\
\hline & 5 & $0,765 \mathrm{a}$ & $0,776 \mathrm{a}$ & $0,794 \mathrm{a}$ & $0,728 \mathrm{a}$ & $0,544 \mathrm{a}$ & $0,686 \mathrm{a}$ & $0,690 \mathrm{a}$ & $0,529 \mathrm{a}$ & $0,681 \mathrm{a}$ & & & $0,674 \mathrm{a}$ & $0,541 \mathrm{a}$ & 15,7 \\
\hline & 6 & $0,769 \mathrm{a}$ & $0,771 \mathrm{a}$ & $0,781 \mathrm{a}$ & $0,687 \mathrm{ab}$ & $0,602 \mathrm{~b}$ & & $0,744 \mathrm{ab}$ & & $0,681 \mathrm{ab}$ & & & $0,697 \mathrm{ab}$ & $0,682 \mathrm{ab}$ & 8,4 \\
\hline & 7 & $0,717 \mathrm{a}$ & $0,746 \mathrm{a}$ & $0,754 \mathrm{a}$ & & $0,631 \mathrm{a}$ & & $0,646 \mathrm{a}$ & & $0,610 \mathrm{a}$ & & & $0,704 \mathrm{a}$ & $0,653 \mathrm{a}$ & 10,1 \\
\hline & 8 & $0,725 \mathrm{a}$ & 0,757 a & $0,771 \mathrm{a}$ & & 0,579 a & & $0,662 \mathrm{a}$ & & 0,645 a & & & 0,667 a & $0,698 \mathrm{a}$ & 13,4 \\
\hline & 9 & $0,758 \mathrm{a}$ & $0,760 \mathrm{a}$ & $0,778 \mathrm{a}$ & & 0,708 a & & 0,696 a & & 0,687 a & & & 0,729 a & $0,713 \mathrm{a}$ & 5,3 \\
\hline & 10 & $0,723 \mathrm{a}$ & $0,754 \mathrm{a}$ & $0,775 \mathrm{a}$ & & $0,683 \mathrm{a}$ & & 0,676 a & & 0,668 a & & & $0,700 \mathrm{a}$ & $0,677 \mathrm{a}$ & 7,8 \\
\hline & 11 & $0,745 \mathrm{a}$ & $0,756 \mathrm{a}$ & $0,761 \mathrm{a}$ & & $0,690 \mathrm{a}$ & & 0,662 a & & 0,656 a & & & $0,716 \mathrm{a}$ & $0,669 \mathrm{a}$ & 7,4 \\
\hline
\end{tabular}

Médias seguidas de mesma letra na linha não diferem significativamente a 5\% de probabilidade pelo teste de Tukey.

${ }^{1 /} \mathrm{DAT}=$ dia após a aplicação do tratamento. 

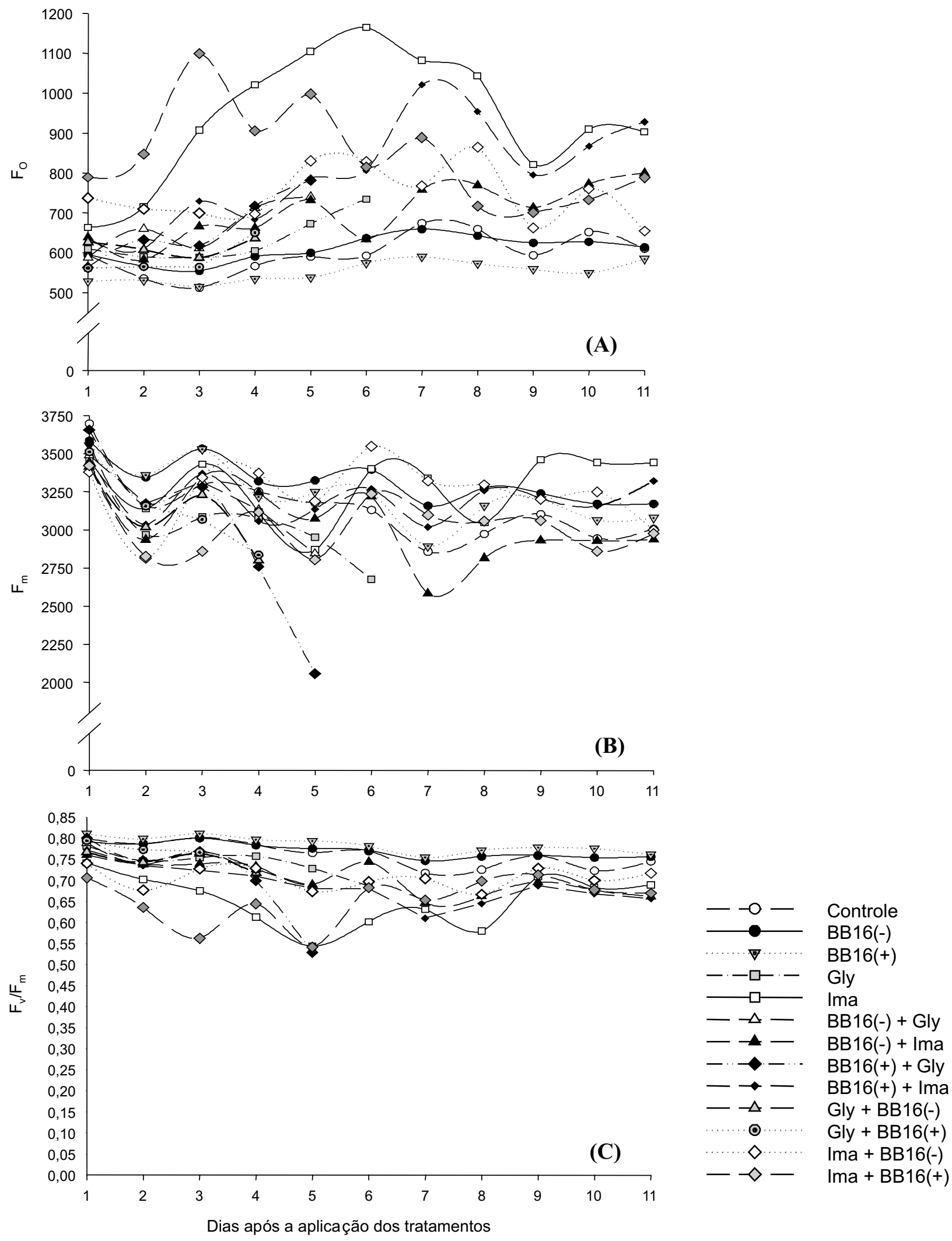

Figura 3 - Valores médios dos parâmetros fotossintéticos mensurados às $16 \mathrm{~h}$ ao longo do período experimental em mudas de Eucalyptus grandis. A $-F_{o}$ (fluorescência inicial), $\mathrm{B}-F_{m}$ (fluorescência máxima) e C $-F_{v} / F_{m}$ (eficiência quântica do fotossistema II). 
necrose total das plantas a partir do sétimo DAT, enquanto sob aplicação prévia ou posterior de $\mathrm{BB} 16$ (em ambas as concentrações) esse evento ocorreu a partir do sexto e quinto DAT, respectivamente. Ou seja, a utilização de BB16 provavelmente acentuou o efeito herbicida conferido pelo glyphosate, uma vez que, sob a aplicação sequenciada desses produtos, ocorreu necrose total das mudas de eucalipto anteriormente às mudas que receberam somente a aplicação do inibidor da EPSPs (3-enoilpiruvilxiquimato-5-fosfato sintase).

Quando se aplicou imazapyr, verificou-se que ao término do período experimental as mudas submetidas à interação imazapyr-BB16 apresentaram sintomas visuais díspares das que receberam glyphosate, não diferindo dos tratamentos controle, BB16(-) e BB16(+).

Quando os herbicidas foram associados à aplicação do análogo de brassinosteroide (BB16), os efeitos de intoxicação foram menos expressivos sob a utilização de imazapyr. No entanto, pôde ser verificado que a aplicação dos herbicidas inibidores de aminoácidos, imazapyr e glyphosate, resultou em efeitos deletérios indiretos no processo fotossintético, mesmo antes da verificação dos sintomas visuais de toxidez. No entanto, a partir do sétimo DAT, somente as mudas que receberam a aplicação de imazapyr (associado ou não a BB16) apresentaram recuperação do aparato fotossintético.

Novos estudos devem ser realizados com o intuito de verificar a interação existente entre as moléculas de BB16 e glyphosate, dada a potencialidade de efeito herbicida da conjunção destas.

\section{AGRADECIMENTOS}

À professora e pesquisadora Dra. Miriam Nuñez-Vázquez, do Departamento de Fisiologia e Bioquímica Vegetal, Instituto Nacional de Ciências Agrícolas (INCA), por ceder o análogo espirostâmico da castasterona.

\section{LITERATURA CITADA}

ARAUS, J. L.; HOGAN, K. P. Comparative leaf structure and patterns of photoinhibition of the neotropical palms. Scheelea zonensis and Socratea durissima growing in clearing and forest understory during the dry season in Panama. Am. J. Bot., v. 81, n. 6, p. 726-738, 1994.
BAKER, N. R.; ROSENQVIST, E. Applications of chlorophyll fluorescence can improve crop production strategies: an examination of future possibilities. J. Exper. Bot., v. 55, n. 403, p. 1607-1621, 2004

BARBAGALLO, R. P. et al. Rapid, noninvasive screening for perturbations of metabolism and plant growth using chlorophyll fluorescence imaging. Plant Physiol., v. 132, n. 2, p. $485-496,2003$.

BOLHÀR-NORDENKAMPH, H. R. et al. Chlorophyll fluorescence as a probe of the photosynthetic competence of leaves in the field: a review of current instrumentation. Funct. Ecol., v. 3, n. 4, p. 497-514, 1989

CATUNDA, M. G. et al. Efeitos de herbicidas na atividade fotossintética e no crescimento de abacaxi (Ananas comossus). Planta Daninha, v. 23, n. 1, p. 115-121, 2005.

CHRISTENSEN, M. G.; TEICHER, H. B.; STREIBIG, J. C. Linking fluorescence induction curve and biomass in herbicide screening. Pest Manag. Sci., v. 59, n. 12, p. 1303-1310, 2003.

CLARK, R. B. Characterization of phosphatase of intact maize roots. J. Agric. Food Chem., v. 23, n. 3, p. 458-460, 1975.

COLL, M. F. et al. Polyhydroxyspirostanones as plant growth regulators. PCT Int. Appl. CO 7J 71/100, AOIN 45/ 00 WO 97/13780, 1995

IRELAND, C. R.; PERCIVAL, M. P.; BAKER, N. R. Modification of the induction of photosynthesis in wheat by glyphosate, an inhibitor of amino acid metabolism. J. Exper. Bot., v. 37, n. 176, p. 299-308, 1986.

KRAUSE, G. H.; WINTER, K. Photoinhibition of photosynthesis in plants growing in natural tropical forest gaps: a chlorophyll fluorescence study. Bot. Acta, v. 109, n. 6, p. 456-462, 1996.

KRISHNA, P. Brassinosteroid-mediated stress responses. J. Plant Growth Regul., v. 22, n. 4, p. 289-297, 2003.

MADSEN, K. H. et al. Photosynthetic parameters in glyphosate-treated sugar-beet (Beta vulgaris L.). Weed Res., v. 32, n. 1, p. $81-88,1995$.

MAXWELL, K.; JOHNSON, G. N. Chlorophyll fluorescence: a practical guide. J. Exper. Bot., v. 51, n. 345, p. 659-668, 2000.

ÖZDEMIR, F. et al. Effects of 24-epibrassinolide on seed germination, seedling growth, lipid peroxidation, proline content and antioxidative system of rice (Oryza sativa L.) under salinity stress. Plant Growth Regul., v. 42, n. 3, p. 203-211, 2004.

RASCHER, U.; LIEBIG, M.; LÜTTGE, U. Evaluation of instant light-responses curves of chlorophyll parameters obtained with a portable chlorophyll fluorometer on site in the field. Plant Cell Environ., v. 23, n. 12, p. 1397-1405, 2000. 
SCHREIBER, U. et al. Measurement of chlorophyll fluorescence within leaves using a modified PAM fluorometer with a fiber-optic microprobe. Photosynth. Res., v. 47, n. 1, p. 103-109, 1996.

STRASSER, R. J.; TSIMILLI-MICHAEL, M.;

SRIVASTAVA, A. The fluorescence transient as a tool to characterize and screen photosynthetic samples. In: YUNUS, M.; PATHER, U.; MOHANLY, P. (Eds.). Probing

photosynthesis: mechanisms, regulation and adaptation. London: Taylor and Francis, 2000. p. 445-483.
XIA, X. J. et al. Pesticides-induced depression of photosynthesis was alleviated by 24-epibrassinolide pretreatment in Cucumis sativus L. Pest. Biochem. Physiol., v. 86, n. 1, p. 42-48, 2006.

YOUNG, A. L.; FRANK, H. A. Energy transfer reactions involving carotenoids: quenching of chlorophyll fluorescence. J. Photochem. Photobiol. B: Biol., v. 36, n. 1, p. 3-15, 1996. 